\title{
Neurophysiological correlates of embodiment and motivational factors during the perception of virtual architectural environments
}

\author{
Giovanni Vecchiato $^{1}$ - Andrea Jelic ${ }^{2}$ Gaetano Tieri ${ }^{3,4}$ - Anton Giulio Maglione ${ }^{1}$. \\ Federico De Matteis $^{2} \cdot$ Fabio Babiloni $^{5}$
}

Published online: 31 July 2015

(C) Marta Olivetti Belardinelli and Springer-Verlag Berlin Heidelberg 2015

\begin{abstract}
The recent efforts aimed at providing neuroscientific explanations of how people perceive and experience architectural environments have largely justified the initial belief in the value of neuroscience for architecture. However, a systematic development of a coherent theoretical and experimental framework is missing. To investigate the neurophysiological reactions related to the appreciation of ambiances, we recorded the electroencephalographic (EEG) signals in an immersive virtual reality during the appreciation of interior designs. Such data have been analyzed according to the working hypothesis that appreciated environments involve embodied simulation mechanisms and circuits mediating approaching stimuli. EEG recordings of 12 healthy subjects have been performed during the perception of three-dimensional interiors that have been simulated in a CAVE system and judged according to dimensions of familiarity, novelty, comfort, pleasantness, arousal and presence. A correlation analysis on personal judgments returned that scores of novelty, pleasantness and comfort are positively correlated, while familiarity and novelty are in negative way. Statistical spectral maps reveal that pleasant, novel
\end{abstract}

Giovanni Vecchiato

giovanni.vecchiato@uniroma1.it

1 Department of Physiology and Pharmacology, University of Rome Sapienza, Rome, Italy

2 Department of Architecture and Design, University of Rome Sapienza, Rome, Italy

3 Department of Psychology, University of Rome Sapienza, Rome, Italy

4 IRCCS Fondazione Santa Lucia, Rome, Italy

5 Department of Molecular Medicine, University of Rome Sapienza, Rome, Italy and comfortable interiors produce a de-synchronization of the mu rhythm over left sensorimotor areas. Interiors judged more pleasant and less familiar generate an activation of left frontal areas (theta and alpha bands), along an involvement of areas devoted to spatial navigation. An increase in comfort returns an enhancement of the theta frontal midline activity. Cerebral activations underlying appreciation of architecture could involve different mechanisms regulating corporeal, emotional and cognitive reactions. Therefore, it might be suggested that people's experience of architectural environments is intrinsically structured by the possibilities for action.

Keywords Electroencephalography · Virtual reality . Architecture $\cdot$ Embodiment $\cdot$ Spatial navigation

\section{Introduction}

Despite several evidence that physical features of built environments in which we live and work influence our psychophysiological states (Lindal and Hartig 2013; Stamps 1999), a systematic research on the cerebral networks activated by perception and appreciation of architectural elements is missing. In this regard, neuroscientific findings could contribute to improve the design of buildings and help to create environments satisfying man's demands. A key cerebral response for the appreciation of art and architecture consists in the activation of the motor system through embodied mechanisms encompassing the simulation of actions, emotions and corporeal sensations (Freedberg and Gallese 2007). Moreover, observation of artworks may be also accompanied by activations of reward-related orbitofrontal areas and cognitive categorization-related prefrontal regions (Sbriscia-Fioretti et al. 
2013), indicating the involvement of emotional and cognitive dimensions.

The involvement of non-overt bodily reactions to the perception and experience of architectural elements can be traced back to early Einfühlung theories, suggesting that the observation of architectural forms may lead to corporeal responses and instantiate a relationship between the esthetic and emotional dimensions as well as body engagement with space (Mallgrave and Ikonomou 1994). To date, such hypotheses have been having a positive validation thanks to recent neuroscientific findings highlighting the crucial role of sensorimotor areas in the appreciation of artworks (Kawabata and Zeki 2004; Sbriscia-Fioretti et al. 2013; Umilta et al. 2012). Elegantly, Freedberg and Gallese (2007) propose a theoretical framework established on the role of embodied simulation and empathy in the esthetic experience of art as tactile sensations, implied gestures and actions. The appreciation and intention to interact with the built environment could also involve motivational factors. In fact, the perception of visual artworks (Sbriscia-Fioretti et al. 2013) and environments featured by curvilinear contours (Vartanian et al. 2013) activates reward circuits formed by medial orbitofrontal and anterior cingulate cortices (Lacey et al. 2011; Vartanian and Goel 2004). Moreover, the activation of left prefrontal areas also emerges during the esthetic judgment (Cela-Conde et al. 2004; Jacobsen et al. 2006; SbrisciaFioretti et al. 2013). These results could be also explained through the affective theoretical framework considering the frontal left hemisphere as a cerebral region responsible for approach-oriented and motivational tendencies (Coan and Allen 2003).

In order to investigate the neurophysiological reactions related to the appreciation of ambiances, we performed electroencephalographic (EEG) recordings in an immersive virtual reality during the appreciation of interior designs. With the working hypothesis that appreciated environments involve embodied simulation mechanisms and circuits mediating approaching stimuli, we compared the spectral activity of the EEG according to a set of subjective dimensions describing the perception of interior designs.

\section{Methods}

\section{Experimental paradigm}

Three-dimensional environments have been simulated in a virtual reality CAVE system formed by three back-projected active stereo screens and a front-projected screen on the floor surrounding the subject (Sanchez-Vives and Slater 2005). Three rooms have been designed in real size $(5 \times 5 \mathrm{~m})$ and tested with different interior designs: empty, modern and cutting-edge furniture shown in Fig. 1. EEG recordings of 12 healthy subjects $(26.8 \pm 2.4$ years $)$ have been performed during the perception of each room, presented in counterbalanced manner for $4 \mathrm{~min}$, during which we asked them to visually explore the environment in which they were immersed. Afterward, the enrolled volunteers expressed judgments for each virtual scenario about familiarity, novelty, comfort, pleasantness, arousal and presence on a 9-point Likert scales (1 indicating the lowest degree of perception, 9 the maximum). Before the stimuli presentation, each subject experienced a familiarization period within an additional virtual environment simulating a laboratory. This experience ended when the subjects said to feel the sense of presence in the environment. The related EEG activity was not used for the further analysis.

\section{Electroencephalographic recordings and signal processing}

The EEG activity was recorded by means of a portable 24-channel system (BEmicro, EBneuro, Italy). Nineteen electrodes were disposed according to the 10-20 I.S., impedances kept below $10 \mathrm{k} \Omega$ and signals acquired at a sampling rate of $256 \mathrm{~Hz}$. Raw EEG traces have been bandpass-filtered ( $\mathrm{hp}=0.5 \mathrm{~Hz}$; lp $=45 \mathrm{~Hz}$ ), and the independent component analysis (ICA) was then applied to detect and remove components due to eye movements. EEG data were grouped to take into account the perception of the three ambiances and further segmented into one-second epochs. Later, the common average reference (CAR) and the power spectral density (PSD) have been computed for each epoch. Individual alpha frequency (IAF) has been calculated for each subject according to individually defined bands and widths (Doppelmayr et al. 1998), focusing the analysis in the theta (IAFx0.4, IAFx0.8), alpha (IAFx0.8, IAFx1.2) and mu (IAF, IAFx1.2) ranges for the present analysis.

\section{Statistical analysis}

Subjectively $z$-scored transformed behavioral scores underwent Pearson's correlation analysis to investigate the relationship among perceptual dimensions used to describe the subjects' experience in the different ambiances. Such behavioral $z$-scores have been also exploited to create two groups of PSDs for each behavioral dimension. Hence, positive $(z>0)$ and negative $(z<0)$ judgments served to contrast the related neurophysiological data, i.e., interiors rated as more novel against the ones rated as less novel, by means of independent sample $t$ test $(p<0.05)$ and False Discovery Rate corrected to prevent statistical error I type (Vecchiato et al. 2010). Results are shown for the theta, alpha and mu bands in the form of statistical topographic maps. 
Fig. 1 Rendering of the three interiors that were used as stimuli, counterbalanced across subjects. Each subject was placed in the middle of the room during the experiment

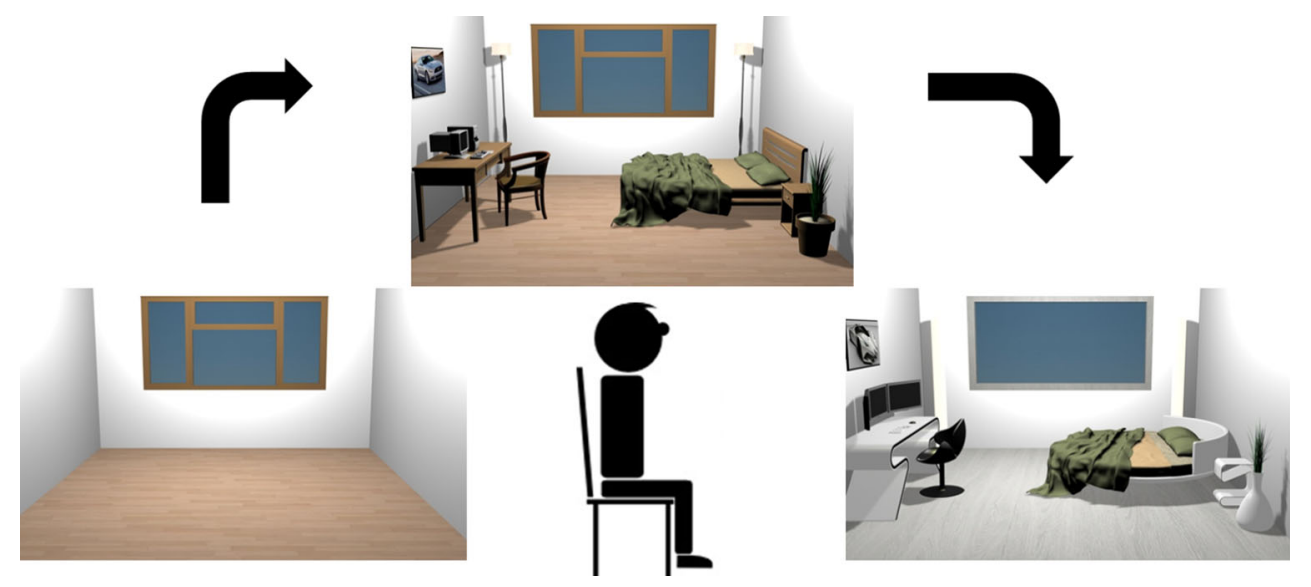

\section{Results}

\section{Behavioral results}

The computation of Pearson's coefficients showed a degree of correlation among some behavioral dimensions, shown in Table 1. In particular, judgments of novelty are positively correlated with pleasantness $(R=0.68, p<0.01)$ and negatively with familiarity $(R=-0.52, p<0.01)$; judgments of pleasantness are also positively correlated with arousal $(R=0.63, p<0.01)$ and comfort $(R=0.42$, $p<0.05)$. Finally, dimension of comfort is also positively correlated with presence $(R=0.40, p<0.05)$.

\section{Topographical patterns of power spectral density}

Statistical spectral maps of pleasantness, related to the perception of highly rated interiors, returned a significant increase in PSD across left frontal and right parieto-occipital regions in the theta band. The same condition also generates a de-synchronization of left frontal and parietal areas in the alpha band as well as a de-synchronization of the mu rhythm across the left sensorimotor areas. Similarly, the dimension of novelty showed a de-synchronization of the mu rhythm across left sensorimotor region for interiors judged more novel. Instead, the analysis of the familiarity dimension revealed the de-synchronization of the alpha band in left orbitofrontal areas associated with less familiar interiors. Finally, the perception of interiors with high comfort rate produced an enhancement of theta frontal midline activity, accompanied by a de-synchronization of the alpha and mu rhythm across right frontal and left sensorimotor areas, respectively. These spectral activations are depicted in Fig. 2. Dimensions of arousal and presence returned no significant activations in these bands.

\section{Discussion}

The analysis of behavioral responses returned that the appreciation of interiors can be described by judgments of novelty, familiarity and comfort. In turn, topographical maps of PSD show the perception of pleasant, novel and comfortable interiors activating the sensorimotor regions, thus suggesting the involvement of embodied simulation mechanisms, as experienced during the appreciation of artworks (Sbriscia-Fioretti et al. 2013; Umilta et al. 2012). Moreover, the asymmetric activation of left frontal regions, related to pleasantness and familiarity, could show the engagement of motivational factors regulating approach (Coan and Allen 2003) already reported during esthetic judgment (Jacobsen et al. 2006). In addition, the enhance of the theta frontal midline activity describing comfort could be related to internalized attention and positive emotional experience (Aftanas and Golocheikine 2001).
Table 1 Pearson's correlation coefficients among behavioral dimensions

\begin{tabular}{lccccc}
\hline & Novelty & Comfort & Pleasantness & Arousal & Presence \\
\hline Familiarity & $-0.52^{* *}$ & 0.08 & -0.06 & -0.07 & 0.11 \\
Novelty & & 0.27 & $0.68^{* *}$ & 0.31 & 0.18 \\
Comfort & & $0.43^{*}$ & 0.19 & $0.40^{*}$ \\
Pleasantness & & & $0.63^{* *}$ & 0.21 \\
Arousal & & & & 0.12 \\
\hline
\end{tabular}

$* p<0.05 ; * * p<0.01$, corrected for multiple comparisons 
Fig. 2 Statistical topographic maps of PSD values highlighting contrast of cerebral activity along the dimensions of pleasantness, novelty, familiarity and comfort (columns) in the theta, alpha and mu frequency bands (rows). Red (blue) color codes increase in PSD during the perception of positively (negatively) rated rooms ( $p<0.05, t$ test, False Discovery Rate corrected) (color figure online)

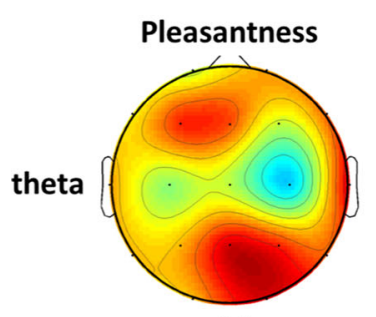

Novelty

Familiarity Comfort
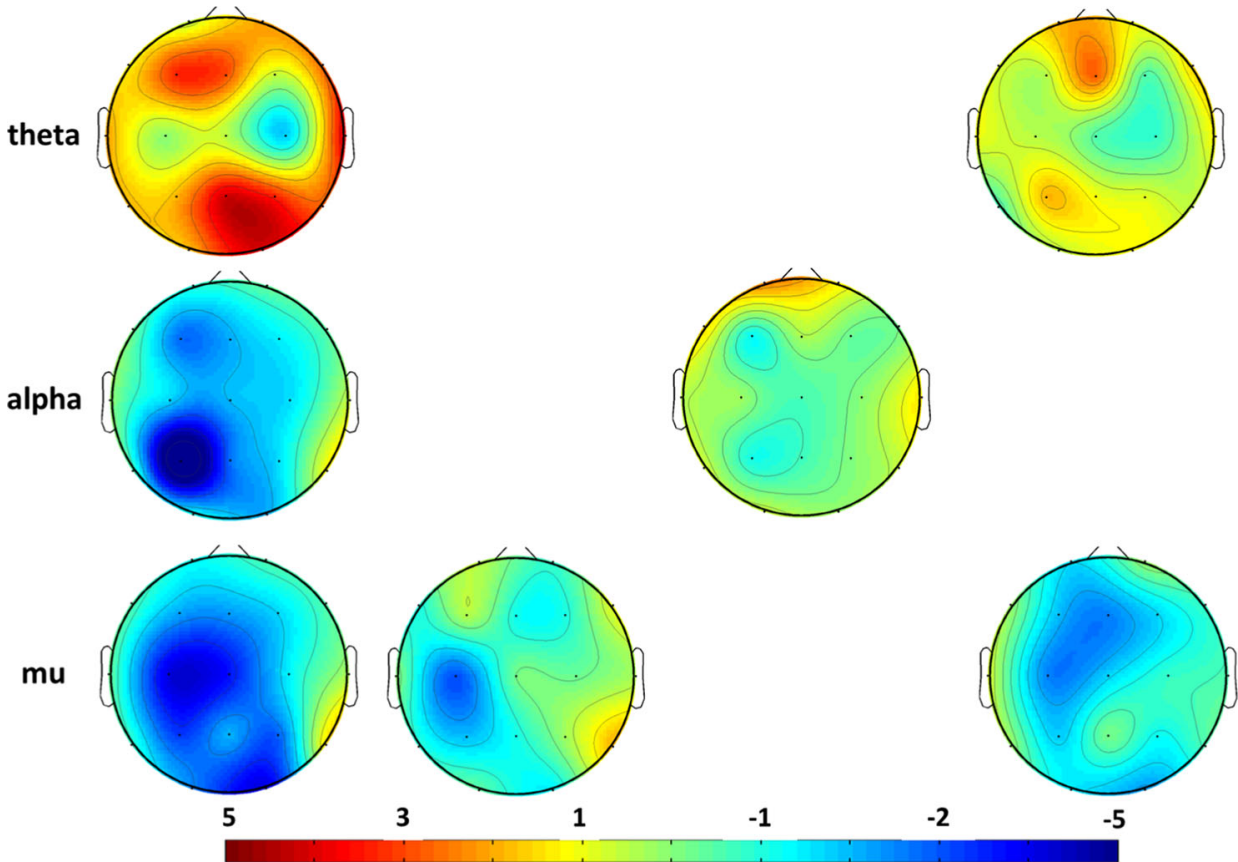

Positively rated rooms > Negatively rated rooms
Negatively rated rooms > Positively rated rooms
Finally, the activity in parietal sites related to pleasantness could reflect the participation in perception of goal-directed hand actions (Salmi et al. 2014) as well as a manifestation of positive moods (Greene et al. 2014).

Starting from the premise that architects design affordances, it might be suggested that people's experience of architectural environments is intrinsically structured by the possibilities for action, which is informed from both sensorimotor knowledge and motivational factors of every individual. Therefore, the cerebral activations underlying the esthetic appreciation of architecture could involve, at the same time, different mechanisms and brain circuits regulating corporeal, emotional and cognitive reactions. Moreover, such neurophysiological (but also behavioral) manifestations could be the representation of the visual and affective impact of past sensations (Barrett and Bar 2009).

The achieved results highlight the simultaneous involvement of different brain mechanisms underlying corporeal, emotional and cognitive reactions. Such findings could have a role in elucidating some of the underlying mechanisms that explain how systematic variations in architectural features lead to behavioral outcomes. These observations could contribute to the development of quantifiable neural markers for testing how the design process of architectural environments can match the changing needs of man, optimizing the design of spaces and possibly improving health.
Acknowledgments This work is partially supported by the grant code C26N149PK8 from Sapienza University of Rome to G.V.

\section{References}

Aftanas L, Golocheikine S (2001) Human anterior and frontal midline theta and lower alpha reflect emotionally positive state and internalized attention: high-resolution EEG investigation of meditation. Neurosci Lett 310:57-60. doi:10.1016/S03043940(01)02094-8

Barrett LF, Bar M (2009) See it with feeling: affective predictions during object perception. Philos Trans R Soc Lond B Biol Sci 364:1325-1334. doi:10.1098/rstb.2008.0312

Cela-Conde CJ, Marty G, Maestú F, Ortiz T, Munar E, Fernández A, Roca M, Rosselló J, Quesney F (2004) Activation of the prefrontal cortex in the human visual aesthetic perception. Proc Natl Acad Sci USA 101:6321-6325. doi:10.1073/pnas. 0401427101

Coan JA, Allen JJB (2003) Frontal EEG asymmetry and the behavioral activation and inhibition systems. Psychophysiology 40:106-114. doi:10.1111/1469-8986.00011

Doppelmayr M, Klimesch W, Pachinger T, Ripper B (1998) Individual differences in brain dynamics: important implications for the calculation of event-related band power. Biol Cybern 79:49-57. doi:10.1007/s004220050457

Freedberg D, Gallese V (2007) Motion, emotion and empathy in esthetic experience. Trends Cogn Sci 11:197-203. doi:10.1016/j. tics.2007.02.003

Greene CM, Flannery O, Soto D (2014) Distinct parietal sites mediate the influences of mood, arousal, and their interaction on human recognition memory. Cogn Affect Behav Neurosci 14:1327-1339. doi:10.3758/s13415-014-0266-y 
Jacobsen T, Schubotz RI, Höfel L, Cramon DYV (2006) Brain correlates of aesthetic judgment of beauty. Neuroimage 29:276-285. doi:10.1016/j.neuroimage.2005.07.010

Kawabata H, Zeki S (2004) Neural correlates of beauty. Neurophysiology 91:1699-1705. doi:10.1152/jn.00696.2003

Lacey S, Hagtvedt H, Patrick VM, Anderson A, Stilla R, Deshpande G, Hu X, Sato JR, Reddy S, Sathian K (2011) Art for reward's sake: visual art recruits the ventral striatum. Neuroimage 55:420-433. doi:10.1016/j.neuroimage.2010.11.027

Lindal PJ, Hartig T (2013) Architectural variation, building height, and the restorative quality of urban residential streetscapes. J Environ Psychol 33:26-36. doi:10.1016/j.jenvp.2012.09.003

Mallgrave HF, Ikonomou E (1994) Empathy, form, and space: Problems in German aesthetics, 1873-1893. Getty Center for the History of Art and the Humanities, Santa Monica

Salmi J, Glerean E, Jääskeläinen IP, Lahnakoski JM, Kettunen J, Lampinen J, Tikka P, Sams M (2014) Posterior parietal cortex activity reflects the significance of others' actions during natural viewing. Hum Brain Mapp 35:4767-4776. doi:10.1002/hbm. 22510

Sanchez-Vives MV, Slater M (2005) From presence to consciousness through virtual reality. Nat Rev Neurosci 6:332-339. doi:10. $1038 /$ nrn 1651
Sbriscia-Fioretti B, Berchio C, Freedberg D, Gallese V, Umiltà MA (2013) ERP Modulation during observation of abstract paintings by Franz Kline. PLoS ONE 8:e75241. doi:10.1371/journal.pone. 0075241

Stamps AE (1999) Physical determinants of preferences for residential facades. Enviro Behav 31:723-751. doi:10.1177/ 00139169921972326

Umilta MA, Berchio C, Sestito M, Freedberg D, Gallese V (2012) Abstract art and cortical motor activation: an EEG study. Front Hum Neurosci 6:311. doi:10.3389/fnhum.2012.00311

Vartanian O, Goel V (2004) Neuroanatomical correlates of aesthetic preference for paintings. Neuroreport 15:893-897

Vartanian O, Navarrete G, Chatterjee A, Fich LB, Leder H, Modroño C, Nadal M, Rostrup N, Skov M (2013) Impact of contour on aesthetic judgments and approach-avoidance decisions in architecture. Proc Natl Acad Sci USA 110:10446-10453. doi:10. 1073/pnas. 1301227110

Vecchiato G, Fallani FDV, Astolfi L, Toppi J, Cincotti F, Mattia D, Salinari S, Babiloni F (2010) The issue of multiple univariate comparisons in the context of neuroelectric brain mapping: an application in a neuromarketing experiment. J Neurosci Methods 191:283-289. doi:10.1016/j.jneumeth.2010.07.009 